\title{
Bose-Einstein Condensation and Free DKP field
}

\author{
R. Casana, V. Ya. Fainberg*, B.M. Pimentel and J. S. Valverde \\ Instituto de Física Teórica, Universidade Estadual Paulista \\ Rua Pamplona 145, CEP 01405-900, São Paulo, SP, Brazil
}

\begin{abstract}
The thermodynamical partition function of the Duffin-Kemmer-Petiau theory is evaluated using the imaginary-time formalism of quantum field theory at finite temperature and path integral methods. The DKP partition function displays two features: (i) full equivalence with the partition function for charged scalar particles and charged massive spin 1 particles; and (ii) the zero mode sector which is essential to reproduce the well-known relativistic Bose-Einstein condensation for both theories.
\end{abstract}

Dedicated to Professor Sílvio Roberto de Azevedo Salinas on the occasion of his 60th birthday.

\section{Introduction}

Bose-Einstein condensation [1, 2] (BEC) is a phenomenon long associated to the liquid helium studies $\left({ }^{4} \mathrm{He}\right.$ and ${ }^{3} \mathrm{He}-{ }^{4} \mathrm{He}$ mixtures). However, recent research deals with BEC shown that it touches several areas of modern physics [3]: in thermodynamics [4 BEC occurs as a phase transition from gas to a new state of matter, quantum mechanics view $\mathrm{BEC}$ as a matter-wave coherence [5] arising from the overlapping de Broglie waves of the atoms and draw an analogy between conventional and "atom lasers" [6, quantum statistics explain BEC as more than one atom sharing a phase space cell, in the quantum theory of atomic traps [7] many atoms condense to the ground state of the trap, in quantum field theory BEC is commonly related to spontaneous symmetry breaking [8, 9, 10.

In quantum field theory BEC the complex scalar field was and is used for studying the thermodynamical properties of physical systems composite of bosonic particles with spin 0 . However, at zero temperature, there is an alternative way to study not only the properties for complex scalar field but also the complex vectorial field that it is known as the massive Duffin-Kemmer-Petiau (DKP) theory [11].

At zero temperature, one important question concerning DKP theory is about the equivalence or not between its spin 0 and 1 sectors and the theories based on the second order Klein-Gordon (KG) and Proca equations, respectively. From the beginning of the 50's the belief on this equivalence was perhaps the principal reason for the abandon of DKP equation in favor of KGF and Proca ones. However, in the 70's this supposed equivalence began to be investigated in several situations involving breaking of symmetries and hadronic processes, showing that in some cases DKP and KG theories can give different results (for a historical review of the development of DKP theory until the decade of 70's see the reference [12]). Moreover, DKP theory follows to be richer than the KG one with respect to the introduction of interactions. In this context, alternative DKP-based models were proposed for the study of meson-nucleus interactions, yielding a better adjustment to the experimental data when compared to the KG-based theory 13. In the same direction, and guided for the formal

*Permanent address: P. N. Lebedev Institute of Physics, Moscow, Russia 
analogy with Dirac equation, approximation techniques formerly developed in the context of nucleon-nucleus scattering were generalized, giving a good description for experimental data of meson-nucleus scattering. The deuteron-nucleus scattering was also studied using DKP, motivated by the fact that this theory suggests a spin 1 structure from combining two spin $\frac{1}{2}$ [14] 15.

Recently there have been a renewed interest in DKP theory. For instance, it has been studied in the context of QCD [16], covariant hamiltonian dynamics [17, in the causal approach [18, in the context of five-dimensional galilean covariance [19], in the scattering $K^{+}$-nucleus [20, in curved space-times [21, 22, 23] 24], etc.

These examples, among others in literature, in some cases break the equivalence between the theories based on DKP and KG/Proca equations, such as in [13] or in Riemann-Cartan space-times [23, 24]. Nevertheless, the question about the equivalence or not still lacks a complete answer nowadays.

As above mentioned, all accomplished studies on the DKP theory were made at zero temperature. The aim of this work is to study the thermodynamics and Bose-Einstein condensation for the massive charged particles by using the DKP theory. In section 2 we present the massive DKP theory in the Minkowski space-time and we make a résumé of the constraint analysis of the model which can be seen in [25, where it has been shown that all the photon Green's functions coincide in DKP and KGF theories. In the section 3 we introduce the partition function and the generating functional of the correlation functions of the theory, and we specialize, separately, the spin 0 and spin 1 sector for the explicit calculation of the respective partition functions. We also analyze explicitly the zero mode contribution in both cases. In the section 4 we gives our conclusions and perspectives.

\section{The Duffin-Kemmer-Petiau theory}

The Duffin-Kemmer-Petiau equation [1] in Minkowski space-time is given by

$$
i \beta^{\mu} \partial_{\mu} \psi-m \psi=0
$$

where the matrices $\beta^{\mu}$ obey the DKP algebra,

$$
\beta^{\alpha} \beta^{\mu} \beta^{\nu}+\beta^{\nu} \beta^{\mu} \beta^{\alpha}=\beta^{\alpha} \eta^{\mu \nu}+\beta^{\nu} \eta^{\mu \alpha}
$$

with $\eta^{\mu \nu}$ being the metric tensor of Minkowski space-time with signature $(+---)$. The $\beta^{\mu}$ are singular matrices which have only three irreducible representations of dimensions 1 , 5 and 10 . The first one is trivial, having no physical meaning and the other two correspond to fields of spin 0 and 1 , respectively.

The DKP equation given in (11) is obtained from the following Lagrangian density

$$
\mathcal{L}=\frac{i}{2} \bar{\psi} \beta^{\mu} \partial_{\mu} \psi-\frac{i}{2} \partial_{\mu} \bar{\psi} \beta^{\mu} \psi-m \bar{\psi} \psi
$$

where $\bar{\psi}=\psi^{\dagger} \eta^{0}$ and $\eta^{0}=2\left(\beta^{0}\right)^{2}-1$, with $\left(\eta^{0}\right)^{2}=1$

In [25, the Hamiltonian canonical approach to the DKP theory is developed in the components and the matrix forms. We make a résumé of the matrix form of this analysis to follow. The canonical conjugate momenta are

$$
p_{\psi}=\frac{i}{2} \psi^{\dagger} \beta^{0} \quad, \quad p_{\bar{\psi}}=-\frac{i}{2} \beta^{0} \psi .
$$

The Hamiltonian density is given by

$$
\mathcal{H}=-\frac{i}{2} \bar{\psi} \beta^{k} \partial_{k} \psi+\frac{i}{2} \partial_{k} \bar{\psi} \beta^{k} \psi+m \bar{\psi} \psi
$$


There is a set of second-class constraints

$$
\begin{gathered}
\bar{\theta}=p_{\psi}-\frac{i}{2} \psi^{\dagger} \beta^{0} \quad, \quad \theta=p_{\bar{\psi}}+\frac{i}{2} \beta^{0} \psi, \\
\omega=\mathbf{M}\left(i \beta^{k} \partial_{k} \psi-m \psi\right) \quad, \quad \bar{\omega}=\left(i \partial_{k} \bar{\psi} \beta^{k}+m \bar{\psi}\right) \mathbf{M},
\end{gathered}
$$

where $\mathbf{M}=1-\left(\beta^{0}\right)^{2}$ is a singular matrix.

\section{The Partition function}

The massive DKP Lagrangian density (3) has a global $U(1)$ symmetry

$$
\begin{aligned}
& \psi \rightarrow \psi^{\prime}=e^{i \alpha} \psi \\
& \bar{\psi} \rightarrow \bar{\psi}^{\prime}=e^{-i \alpha} \bar{\psi}
\end{aligned}
$$

whose conserved current is $j^{\mu}=\bar{\psi} \beta^{\mu} \psi$, and the respective conserved charge

$$
Q=\int d^{3} \mathbf{x} j^{0}(x)
$$

Then the partition function in the imaginary-time formalism for the massive DKP field is

$$
\begin{aligned}
& Z=N(\beta) \int \mathcal{D} p_{\psi} \mathcal{D} p_{\bar{\psi}} \int_{\text {periodic }} \mathcal{D} \psi \mathcal{D} \bar{\psi} \delta(\theta) \delta(\bar{\theta}) \delta(\omega) \delta(\bar{\omega}) \\
& \exp \left\{\int_{0}^{\beta} d \tau \int d^{3} \mathbf{x}\left[i p_{\psi} \partial_{\tau} \psi+i \partial_{\tau} \bar{\psi} p_{\bar{\psi}}-\mathcal{H}+\mu j^{0}\right]\right\},
\end{aligned}
$$

where $N(\beta)$ is an infinite normalizing factor which will be determined later [26]. We integrate over periodic DKP field due to its bosonic character.

To compute the correlation functions of the theory we couple the DKP field to external sources $\bar{\eta}$ and $\eta$, thus the generating functional is read

$$
\begin{aligned}
Z[\eta, \bar{\eta}]=N(\beta) \int \mathcal{D} p_{\psi} \mathcal{D} p_{\bar{\psi}} \int_{\text {periodic }} \mathcal{D} \psi \mathcal{D} \bar{\psi} \delta(\theta) \delta(\bar{\theta}) \delta(\omega) \delta(\bar{\omega}) \\
\quad \exp \left\{\int_{0}^{\beta} d \tau \int d^{3} \mathbf{x}\left[i p_{\psi} \partial_{\tau} \psi+i \partial_{\tau} \bar{\psi} p_{\bar{\psi}}-\mathcal{H}+\mu j^{0}+\bar{\eta} \psi+\bar{\psi} \eta\right]\right\} .
\end{aligned}
$$

After the integration on the canonical conjugate momenta, we define the matrix $\beta^{\tau} \equiv i \beta^{0}$; and the constraints $\omega$ and $\bar{\omega}$ can be rewritten as

$$
\omega=\mathbf{M D} \psi \quad, \quad \bar{\omega}=\overline{\mathbf{D} \psi} \mathbf{M}
$$

due the property $\beta^{0} \mathbf{M}=0=\mathbf{M} \beta^{0}$, and we have used a short notation,

$$
\mathbf{D} \psi=i \beta^{a} \partial_{a} \psi-m \psi \quad, \quad \overline{\mathbf{D} \psi}=i \partial_{a} \bar{\psi} \beta^{a}+m \bar{\psi},
$$

where $a=\tau, 1,2,3$. With all definitions noted above, the generating functional (11) appears to be

$$
Z[\eta, \bar{\eta}]=N(\beta) \int_{\text {periodic }} \mathcal{D} \psi \mathcal{D} \bar{\psi} \delta(\mathbf{M} \mathbf{D} \psi) \delta(\overline{\mathbf{D} \psi} \mathbf{M}) e^{S}
$$


where the action $S$ is given by

$$
S=\int_{0}^{\beta} d \tau \int d^{3} \mathbf{x}\left(\frac{1}{2} \bar{\psi} \mathbf{D} \psi-\frac{1}{2} \overline{\mathbf{D} \psi} \psi+\mu \bar{\psi} \beta^{0} \psi+\bar{\eta} \psi+\bar{\psi} \eta\right) .
$$

After the exponentiation of the $\delta$-functions, we use the periodicity condition over the fields to make some integration by parts, and get the following expression for the generating functional

$$
Z[\eta, \bar{\eta}]=N(\beta) \int_{\text {periodic }} \mathcal{D} \psi \mathcal{D} \bar{\psi} \mathcal{D} \rho \mathcal{D} \bar{\sigma} e^{S^{1}}
$$

where the auxiliary fields $\rho$ and $\sigma$ were used to exponentiate the constraints; and the action $S^{1}$ is given by

$$
S^{1}=\int_{0}^{\beta} d \tau \int d^{3} \mathbf{x}\left[\bar{\psi}\left(\mathbf{D}+\mu \beta^{0}\right) \psi-i \overline{\mathbf{D M} \sigma} \psi-i \bar{\psi} \mathbf{D M} \rho+\bar{\eta} \psi+\bar{\psi} \eta\right]
$$

Into the generating functional (15), we make the following change of variables,

$$
\psi \rightarrow \psi+\psi_{s} \quad, \quad \bar{\psi} \rightarrow \bar{\psi}+\bar{\psi}_{s}
$$

such the fields $\psi_{s}$ and $\bar{\psi}_{s}$ satisfy the following equations

$$
\left(\mathbf{D}+\mu \beta^{0}\right) \psi_{s}-i \mathbf{D M} \rho+\eta=0 \quad ; \quad-\overline{\mathbf{D} \psi_{s}}+\mu \bar{\psi}_{s} \beta^{0}-i \overline{\mathbf{D M} \sigma}+\bar{\eta}=0 .
$$

And after it, we carry out another change of variables on the auxiliary fields $\rho$ and $\sigma$,

$$
\bar{\sigma} \rightarrow \bar{\sigma}+\bar{\sigma}_{s} \quad, \quad \rho \rightarrow \rho+\rho_{s}
$$

where the fields $\rho_{s}$ and $\sigma_{s}$ satisfy the equations

$$
m \mathbf{M} \rho_{s}-i \mathbf{M} \eta=0 \quad ; \quad m \bar{\sigma}_{s} \mathbf{M}+i \bar{\eta} \mathbf{M}=0 .
$$

Thus, after some additional calculations in the generating functional in (15), we get

$$
Z[\eta, \bar{\eta}]=\exp \left\{-\int_{0}^{\beta} d \tau \int d^{3} \mathbf{x} \bar{\eta}\left(\mathbf{D}+\mu \beta^{0}\right)^{-1} \eta+\frac{1}{m} \bar{\eta} \mathbf{M} \eta\right\} Z,
$$

where $Z$ is the partition function given in (10) which, after all operations made above, it is read as

$$
Z=N(\beta) \int_{\text {periodic }} \mathcal{D} \psi \mathcal{D} \bar{\psi} \mathcal{D} \rho \mathcal{D} \bar{\sigma} \exp \left[\int_{0}^{\beta} d \tau \int d^{3} \mathbf{x} \bar{\psi}\left(\mathbf{D}+\mu \beta^{0}\right) \psi+m \bar{\sigma} \mathbf{M} \rho\right] .
$$

We can note that the functional integration over the fields $\bar{\sigma}$ and $\rho$ give an infinity quantity, due to singular character of the $\mathbf{M}$-matrix, which is irrelevant to the thermodynamical properties of the physical system. Thus, we can write the partition function as

$$
Z=N_{\text {periodic }}^{\prime} N(\beta) \int_{\mathcal{D}} \mathcal{D} \bar{\psi} \exp \left[\int_{0}^{\beta} d \tau \int d^{3} \mathbf{x} \bar{\psi}\left(\mathbf{D}+\mu \beta^{0}\right) \psi\right] .
$$

The fields may be expanded as a Fourier series [26]

$$
\begin{aligned}
& \psi(\tau, \mathbf{x})=\psi_{\text {zero }}+\frac{1}{\beta} \sum_{n} \int \frac{d^{3} \mathbf{p}}{(2 \pi)^{3}} e^{i\left(\mathbf{p} \cdot \mathbf{x}+\omega_{n} \tau\right)} \psi_{n}(\mathbf{p}) \\
& \bar{\psi}(\tau, \mathbf{x})=\bar{\psi}_{\text {zero }}+\frac{1}{\beta} \sum_{n} \int \frac{d^{3} \mathbf{p}}{(2 \pi)^{3}} e^{i\left(\mathbf{p} \cdot \mathbf{x}+\omega_{n} \tau\right)} \bar{\psi}_{n}(\mathbf{p}),
\end{aligned}
$$


where $\psi_{\text {zero }}$ and $\bar{\psi}_{\text {zero }}$ are independent of $(\tau, \mathbf{x})$ and carry the full infrared character of the DKP field; that is, $\psi_{n=0}(\mathbf{p}=0)=0$ and $\bar{\psi}_{n=0}(\mathbf{p}=0)=0$. And due to bosonic character of DKP field, we impose the constraint of periodicity such $\psi(\tau=0, \mathbf{x})=\psi(\tau=\beta, \mathbf{x})$ for all $\mathbf{x}$, thus the frequency will be $\omega_{n}=2 \pi n / \beta, n= \pm 1, \pm 2, \ldots$.

Substituting (22) into (21), we find

$$
\int_{0}^{\beta} d \tau \int d^{3} \mathbf{x} \bar{\psi}\left(\mathbf{D}+\mu \beta^{0}\right) \psi=\beta V \bar{\psi}_{\text {zero }}\left(-m+\mu \beta^{0}\right) \psi_{\text {zero }}+S^{\prime},
$$

where the free infrared action is

$$
S^{\prime}=-\frac{1}{\beta} \sum_{n} \int \frac{d^{3} \mathbf{p}}{(2 \pi)^{3}} \bar{\psi}_{-n}(-\mathbf{p})\left[i \beta^{0}\left(\omega_{n}+i \mu\right)+\beta^{k} p_{k}+m\right] \psi_{n}(\mathbf{p}) .
$$

The partition function given in (21), can be rewritten using the equation (24) as

$$
Z=N^{\prime} N(\beta) Z_{\text {zero }} Z^{\prime},
$$

where $Z_{z e r o}$ is the zero mode contribution

$$
Z_{\text {zero }}=\exp \left[\beta V \bar{\psi}_{\text {zero }}\left(-m+\mu \beta^{0}\right) \psi_{\text {zero }}\right],
$$

and the $Z^{\prime}$ contribution is

$$
Z^{\prime}=\prod_{n} \prod_{\mathbf{p}}\left[\operatorname{det}\left(i \beta^{0}\left(\omega_{n}+i \mu\right)+\beta^{k} p_{k}+m\right)\right]^{-1} .
$$

\subsection{The spin 0 sector}

It is worth to notice that until this moment we have not used an explicit representation of the DKP algebra. In this point we specialize in the spin 0 sector of DKP theory, thus we calculate every contribution in (25). We first compute the (27) $Z^{\prime}$ partition function, to make it we use an explicit $5 \times 5$ representation (58) (see Appendix) of the DKP algebra, thus we obtain

$$
\ln Z^{\prime}=-V \sum_{n} \int \frac{d^{3} \mathbf{p}}{(2 \pi)^{3}} \ln \left\{m^{3}\left[\left(\omega_{n}+i \mu\right)^{2}+\omega^{2}\right]\right\}
$$

where $\omega=\sqrt{\mathbf{p}^{2}+m^{2}}$, and ignoring the $\beta$-independent constant, we can rewrite (28) to show explicitly the existence of particles and anti-particles, thus

$$
\begin{array}{rl}
\ln Z^{\prime}=2 & V \ln (\beta) \sum_{n} \int \frac{d^{3} \mathbf{p}}{(2 \pi)^{3}}-\frac{V}{2} \sum_{n} \int \frac{d^{3} \mathbf{p}}{(2 \pi)^{3}} \ln \left\{\beta^{2}\left[\omega_{n}^{2}+(\omega-\mu)^{2}\right]\right\}+ \\
& -\frac{V}{2} \sum_{n} \int \frac{d^{3} \mathbf{p}}{(2 \pi)^{3}} \ln \left\{\beta^{2}\left[\omega_{n}^{2}+(\omega+\mu)^{2}\right]\right\} .
\end{array}
$$

To calculate the sum on $n$ in the last two terms we use the standard results founded in the literature [8, 26, thus

$$
\begin{aligned}
\ln Z^{\prime}=- & V \int \frac{d^{3} \mathbf{p}}{(2 \pi)^{3}}\left[\beta \omega+\ln \left(1-e^{-\beta(\omega-\mu)}\right)+\ln \left(1-e^{-\beta(\omega+\mu)}\right)\right]+ \\
& +2 V \ln (\beta) \sum_{n} \int \frac{d^{3} \mathbf{p}}{(2 \pi)^{3}},
\end{aligned}
$$


where the last term is an infinity $\beta$-dependent quantity which has to be cancelled by the $N(\beta)$ constant, thus we set it to be

$$
\ln N(\beta)=-2 V \ln (\beta) \sum_{n} \int \frac{d^{3} \mathbf{p}}{(2 \pi)^{3}}
$$

in total agreement with the literature when is studied the complex or charged scalar field [8, 9, 28.

\section{The zero mode contribution}

Now, we show the existence of the zero mode sector for the spin 0 DKP field. The zero mode equation is obtained from the action given in (21),

$$
\left(\mathbf{D}+\mu \beta^{0}\right) \psi=0
$$

by setting the fields $\psi$ to be independent of $(\tau, \mathbf{x})$, thus

$$
\left(-m+\mu \beta^{0}\right) \psi_{\text {zero }}=0
$$

then the determinant of the matrix $\left(-m+\mu \beta^{0}\right)$ must be set to zero for getting non-trivial solutions,

$$
\operatorname{det}\left(-m+\mu \beta^{0}\right)=-m^{3}\left(m^{2}-\mu^{2}\right)=0 .
$$

It is easy to see that we get non-trivial solutions when the chemical potential $\mu$ reaches the values $\pm m$. The field $\psi_{z e r o}$ is a five complex component column matrix,

$$
\psi_{\text {zero }}=\left(a, b, c^{1}, c^{2}, c^{3}\right)^{T} .
$$

Solving the matrix equation above, we find

$$
c^{k}=0 \quad, \quad a=\frac{\mu b}{m} \quad \text { or } \quad a=\frac{m b}{\mu} .
$$

Then, using the above relations we find that zero mode contribution of the partition function is for $\mu \neq \pm m$

$$
Z_{\text {zero }}=-\beta V\left(m^{2}-\mu^{2}\right) \xi^{2}
$$

with $\xi$ being an arbitrary real parameter.

Finally, the full partition function for the spin 0 sector of DKP field is

$$
\ln Z=-\beta V\left(m^{2}-\mu^{2}\right) \xi^{2}-V \int \frac{d^{3} \mathbf{p}}{(2 \pi)^{3}}\left[\beta \omega+\ln \left(1-e^{-\beta(\omega-\mu)}\right)+\ln \left(1-e^{-\beta(\omega+\mu)}\right)\right]
$$

reproducing exactly the partition function of charged scalar field 8 ] when we take the contribution of the zero mode into account. The momentum integral is convergent only if $|\mu| \leq m$. The parameter $\xi$ that appears in the final expression for the partition function is not determined a priori, it should be treated as an variational parameter which is related to the charge carried by the condensed particles. Then, at fixed $\beta$ and $\mu, \ln Z$ is an extremum with respect to variations of such a free parameter,

$$
\frac{\partial \ln Z}{\partial \xi}=-2 \beta V\left(m^{2}-\mu^{2}\right) \xi=0
$$

which implies that $\xi=0$, unless $|\mu|=m$ and in this case $\xi$ is undetermined by this variational condition. Thus, when $|\mu|<m$ the contribution of the zero mode to the partition function is cancelled. 


\subsubsection{The Bose-Einstein condensation}

By completeness, we briefly summarize [9] some characteristic of Bose-Einstein condensation in the case of a relativistic Bose gas. The question which we make is this: What is the requirement for BEC to take place at relativistic temperatures (i.e., $T \gg m$ ) and what is the nature of the phase transition.

For $|\mu|<m$, the $\xi$ parameter is set to zero in (38), and the charge density is given by

$$
\rho=\frac{1}{\beta V}\left(\frac{\partial \ln Z}{\partial \mu}\right)=\int \frac{d^{3} \mathbf{p}}{(2 \pi)^{3}}\left(\frac{1}{e^{\beta(\omega-\mu)}-1}-\frac{1}{e^{\beta(\omega+\mu)}-1}\right),
$$

as the chemical potential satisfies $|\mu|<m$, thus, the charge densities of particles and antiparticles are non-negative. Note that (40) is really an implicit formula for $\mu$ as a function of $\rho$ and $T$. For $T$ above the some critical temperature $T_{C}$, one can always find a $\mu$ such that (40) holds. If the density $\rho$ is held fixed and the temperature is lowered, $\mu$ will increase until the point $|\mu|=m$ is reached, thus, in the region $T \geq T_{C} \gg m$ we obtain [9]

$$
|\rho| \approx \frac{1}{3} m T^{2}
$$

When $|\mu|=m$ and the temperature is lowered even further such $T<T_{C}$, the charge density is written as

$$
\rho=\frac{1}{\beta V}\left(\frac{\partial \ln Z}{\partial \mu}\right)_{\mu=m}=\rho_{0}+\rho^{*}(\beta, \mu=m),
$$

where $\rho_{0}=2 m \xi^{2}$ is a charge contribution from the condensate (the zero-momentum mode) and the $\rho^{*}(\beta, \mu=m)$ is the thermal particle excitations (finite-momentum modes) which is given by (40) with $|\mu|=m$.

The critical temperature $T_{C}$, in which the Bose-Einstein condensation occurs, corresponds when $|\mu|=m$ is reached and determined implicitly by the equation

$$
\rho=\rho^{*}\left(\beta_{C}, \mu=m\right),
$$

which implies that

$$
T_{C}=\left(\frac{3|\rho|}{m}\right)^{1 / 2}
$$

At temperatures $T<T_{C}$, (42) is an equation for charge density $\rho-\rho_{0}$ of the $\mathbf{p} \neq \mathbf{0}$ states,

$$
\rho-\rho_{0}=\frac{1}{3} m T^{2},
$$

so that the charge density in the ground state $(\mathbf{p}=\mathbf{0})$ is

$$
\rho_{0}=\rho\left(1-\left[\frac{T}{T_{C}}\right]^{2}\right),
$$

as the temperature is lowered the fraction of charge in $\mathbf{p}=\mathbf{0}$ state increase to unity. We note that (44) leads to the important result: the necessary condition for that any ideal Bose gas of mass $m$ Bose-Einstein condense at a relativistic temperature (i. e., $T_{C} \gg m$ ), is that $\rho \gg m^{3}$. Additional literature about Bose-Einstein condensation can be found in [4, 27, 28, 3. 


\subsection{The spin 1 sector}

Now we study spin 1 sector of DKP theory, thus we calculate every contribution in (25). We first compute the (27) $Z^{\prime}$ partition function. For this purpose, we consider an explicit $10 \times 10$ representation (59) (see Appendix) of the DKP algebra, thus we get the following contribution

$$
\ln Z^{\prime}=-V \sum_{n} \int \frac{d^{3} \mathbf{p}}{(2 \pi)^{3}} \ln \left\{m^{4}\left[\left(\omega_{n}+i \mu\right)^{2}+\omega^{2}\right]^{3}\right\}
$$

and more one time by ignoring the $\beta$-independent constant, we can rewrite (47) to show explicitly the existence of particles and anti-particles, thus

$$
\begin{array}{rl}
\ln Z^{\prime}=6 & V \ln (\beta) \sum_{n} \int \frac{d^{3} \mathbf{p}}{(2 \pi)^{3}}-\frac{3 V}{2} \sum_{n} \int \frac{d^{3} \mathbf{p}}{(2 \pi)^{3}} \ln \left\{\beta^{2}\left[\omega_{n}^{2}+(\omega-\mu)^{2}\right]\right\}+ \\
& -\frac{3 V}{2} \sum_{n} \int \frac{d^{3} \mathbf{p}}{(2 \pi)^{3}} \ln \left\{\beta^{2}\left[\omega_{n}^{2}+(\omega+\mu)^{2}\right]\right\} .
\end{array}
$$

Here, we can see the six degree of freedom corresponding to two complex massive vector fields or Proca's complex fields. Carrying out the sum in both last terms and after some manipulations [8, 26], we find

$$
\begin{aligned}
\ln Z^{\prime}=- & 3 V \int \frac{d^{3} \mathbf{p}}{(2 \pi)^{3}}\left[\beta \omega+\ln \left(1-e^{-\beta(\omega-\mu)}\right)+\ln \left(1-e^{-\beta(\omega+\mu)}\right)\right]+ \\
& +6 V \ln (\beta) \sum_{n} \int \frac{d^{3} \mathbf{p}}{(2 \pi)^{3}},
\end{aligned}
$$

the last term is an infinity quantity $\beta$-dependent, and, as in the spin 0 sector, it will be cancelled by the $N(\beta)$ contribution, thus with all security we set it to be

$$
\ln N(\beta)=-6 V \ln (\beta) \sum_{n} \int \frac{d^{3} \mathbf{p}}{(2 \pi)^{3}} .
$$

\section{The zero mode contribution}

In this case, we also show the existence of the zero mode sector for the spin 1 DKP field. The zero mode equation is obtained from the action given in (21),

$$
\left(\mathbf{D}+\mu \beta^{0}\right) \psi=0
$$

by setting the fields $\psi$ to be independent of $(\tau, \mathbf{x})$, thus

$$
\left(-m+\mu \beta^{0}\right) \psi_{\text {zero }}=0
$$

then o determinant of the matrix $\left(-m+\mu \beta^{0}\right)$ must be set to zero for getting non-trivial solutions,

$$
\operatorname{det}\left(-m+\mu \beta^{0}\right)=m^{4}\left(m^{2}-\mu^{2}\right)^{3}=0 .
$$

It is easy to see that we get non-trivial solutions when the chemical potential $\mu$ reaches the values $\pm m$, and in this representation the field $\psi_{\text {zero }}$ is a ten complex component column matrix,

$$
\psi_{\text {zero }}=\left(\psi^{\tau}, \psi^{1}, \psi^{2}, \psi^{3}, \psi^{4}, \psi^{5}, \psi^{6}, \psi^{7}, \psi^{8}, \psi^{9}\right)^{T} .
$$


By solving the above matrix equation, we find

$$
\begin{gathered}
\psi^{\tau}=\psi^{4}=\psi^{5}=\psi^{6}=0 \\
\psi^{1}=\frac{\mu \psi^{7}}{m} \quad, \quad \psi^{2}=\frac{\mu \psi^{8}}{m} \quad, \quad \psi^{3}=\frac{\mu \psi^{9}}{m} .
\end{gathered}
$$

By using the above relations we find that zero mode contribution of the partition function is for $\mu \neq \pm m$

$$
Z_{\text {zero }}=-\beta V\left(m^{2}-\mu^{2}\right)\left(\xi_{1}^{2}+\xi_{2}^{2}+\xi_{3}^{2}\right)
$$

with $\xi_{k}$ being an arbitrary real parameters related to the spatial components to the zero mode DKP field, i.e, $\psi^{1}, \psi^{2}, \psi^{3}$.

Finally, the full partition function for the spin 1 sector of DKP field is

$$
\begin{aligned}
\ln Z=- & \beta V\left(m^{2}-\mu^{2}\right)\left(\xi_{1}^{2}+\xi_{2}^{2}+\xi_{3}^{2}\right)+ \\
& -3 V \int \frac{d^{3} \mathbf{p}}{(2 \pi)^{3}}\left[\beta \omega+\ln \left(1-e^{-\beta(\omega-\mu)}\right)+\ln \left(1-e^{-\beta(\omega+\mu)}\right)\right] .
\end{aligned}
$$

The $\xi_{k}$ parameters are related to the degree of freedom of the spin 1 DKP field, i.e, three degrees of freedom of the massive charged vector field. The zero mode sector is again absolutely necessary to reproduce the BEC as the spin zero sector; however, the critical temperature is the same for every degree of freedom.

\section{Conclusions and remarks}

The free massive DKP theory at Finite Temperature (FT) is equivalent to both complex massive scalar field and complex vector field theories at FT. It reproduces the relativistic Bose-Einstein condensation in both sectors, where we show in a clean and elegant way the zero mode existence and its contribution to BEC.

The perspectives to follow are to study the DKP field coupled to the quantized electromagnetic field and the implementation of the renormalization process at zero temperature which will allow to extend the analysis at Finite temperature. And, Bose-Einstein condensation in curved space-times [29] using the DKP theory minimally coupled. Advances in this directions will be reported elsewhere.

\section{Acknowledgements}

We thank to Profs. L. Tomio and Victo S. Filho for additional references related to the BoseEinstein condensation. This work was supported by FAPESP/Brazil (R.C., full support grant 01/12611-7; V.Ya.F., grant 01/12585-6; B.M.P., grant 02/00222-9, J. V. full support grant 00/03812-6), RFFI/ Russia (V.Ya.F., grant 02-02-16946), LSS-1578.2003.2 (V.Ya.F.), and $\mathrm{CNPq} /$ Brazil (B.M.P.). 


\section{Appendix}

\section{Spin 0 and Spin 1 representation}

We use the following representation for the spin 0 sector of DKP algebra

$$
\begin{aligned}
& \beta^{0}=\left[\begin{array}{lllll}
0 & 1 & 0 & 0 & 0 \\
1 & 0 & 0 & 0 & 0 \\
0 & 0 & 0 & 0 & 0 \\
0 & 0 & 0 & 0 & 0 \\
0 & 0 & 0 & 0 & 0
\end{array}\right] \quad, \quad \beta^{1}=\left[\begin{array}{rrrrr}
0 & 0 & 1 & 0 & 0 \\
0 & 0 & 0 & 0 & 0 \\
-1 & 0 & 0 & 0 & 0 \\
0 & 0 & 0 & 0 & 0 \\
0 & 0 & 0 & 0 & 0
\end{array}\right] \\
& \beta^{2}=\left[\begin{array}{lllll}
0 & 0 & 0 & 1 & 0 \\
0 & 0 & 0 & 0 & 0 \\
0 & 0 & 0 & 0 & 0 \\
-1 & 0 & 0 & 0 & 0 \\
0 & 0 & 0 & 0 & 0
\end{array}\right] \quad, \quad \beta^{3}=\left[\begin{array}{lllll}
0 & 0 & 0 & 0 & 1 \\
0 & 0 & 0 & 0 & 0 \\
0 & 0 & 0 & 0 & 0 \\
0 & 0 & 0 & 0 & 0 \\
-1 & 0 & 0 & 0 & 0
\end{array}\right]
\end{aligned}
$$

and for the spin 1 sector of DKP algebra

$$
\begin{aligned}
& \beta^{0}=\left[\begin{array}{rrrrrrrrrr}
0 & 0 & 0 & 0 & 0 & 0 & 0 & 0 & 0 & 0 \\
0 & 0 & 0 & 0 & 0 & 0 & 0 & 1 & 0 & 0 \\
0 & 0 & 0 & 0 & 0 & 0 & 0 & 0 & 1 & 0 \\
0 & 0 & 0 & 0 & 0 & 0 & 0 & 0 & 0 & 1 \\
0 & 0 & 0 & 0 & 0 & 0 & 0 & 0 & 0 & 0 \\
0 & 0 & 0 & 0 & 0 & 0 & 0 & 0 & 0 & 0 \\
0 & 0 & 0 & 0 & 0 & 0 & 0 & 0 & 0 & 0 \\
0 & 1 & 0 & 0 & 0 & 0 & 0 & 0 & 0 & 0 \\
0 & 0 & 1 & 0 & 0 & 0 & 0 & 0 & 0 & 0 \\
0 & 0 & 0 & 1 & 0 & 0 & 0 & 0 & 0 & 0
\end{array}\right] \quad, \quad \beta^{1}=\left[\begin{array}{rrrrrrrrrr}
0 & 0 & 0 & 0 & 0 & 0 & 0 & -1 & 0 & 0 \\
0 & 0 & 0 & 0 & 0 & 0 & 0 & 0 & 0 & 0 \\
0 & 0 & 0 & 0 & 0 & 0 & -1 & 0 & 0 & 0 \\
0 & 0 & 0 & 0 & 0 & 1 & 0 & 0 & 0 & 0 \\
0 & 0 & 0 & 0 & 0 & 0 & 0 & 0 & 0 & 0 \\
0 & 0 & 0 & -1 & 0 & 0 & 0 & 0 & 0 & 0 \\
0 & 0 & 1 & 0 & 0 & 0 & 0 & 0 & 0 & 0 \\
1 & 0 & 0 & 0 & 0 & 0 & 0 & 0 & 0 & 0 \\
0 & 0 & 0 & 0 & 0 & 0 & 0 & 0 & 0 & 0 \\
0 & 0 & 0 & 0 & 0 & 0 & 0 & 0 & 0 & 0
\end{array}\right] \\
& \beta^{2}=\left[\begin{array}{rrrrrrrrrr}
0 & 0 & 0 & 0 & 0 & 0 & 0 & 0 & -1 & 0 \\
0 & 0 & 0 & 0 & 0 & 0 & 1 & 0 & 0 & 0 \\
0 & 0 & 0 & 0 & 0 & 0 & 0 & 0 & 0 & 0 \\
0 & 0 & 0 & 0 & -1 & 0 & 0 & 0 & 0 & 0 \\
0 & 0 & 0 & 1 & 0 & 0 & 0 & 0 & 0 & 0 \\
0 & 0 & 0 & 0 & 0 & 0 & 0 & 0 & 0 & 0 \\
0 & -1 & 0 & 0 & 0 & 0 & 0 & 0 & 0 & 0 \\
0 & 0 & 0 & 0 & 0 & 0 & 0 & 0 & 0 & 0 \\
1 & 0 & 0 & 0 & 0 & 0 & 0 & 0 & 0 & 0 \\
0 & 0 & 0 & 0 & 0 & 0 & 0 & 0 & 0 & 0
\end{array}\right] \quad, \quad \beta^{3}=\left[\begin{array}{rrrrrrrrrr}
0 & 0 & 0 & 0 & 0 & 0 & 0 & 0 & 0 & -1 \\
0 & 0 & 0 & 0 & 0 & -1 & 0 & 0 & 0 & 0 \\
0 & 0 & 0 & 0 & 1 & 0 & 0 & 0 & 0 & 0 \\
0 & 0 & 0 & 0 & 0 & 0 & 0 & 0 & 0 & 0 \\
0 & 0 & -1 & 0 & 0 & 0 & 0 & 0 & 0 & 0 \\
0 & 1 & 0 & 0 & 0 & 0 & 0 & 0 & 0 & 0 \\
0 & 0 & 0 & 0 & 0 & 0 & 0 & 0 & 0 & 0 \\
0 & 0 & 0 & 0 & 0 & 0 & 0 & 0 & 0 & 0 \\
0 & 0 & 0 & 0 & 0 & 0 & 0 & 0 & 0 & 0 \\
1 & 0 & 0 & 0 & 0 & 0 & 0 & 0 & 0 & 0
\end{array}\right] .
\end{aligned}
$$

\section{References}

[1] S. N. Bose, Z. Phys. 26, 178 (1924).

[2] A. Einstein, Sitz. Ber. Kgl. Preuss. Akad. Wiss. 22, 261 (1924).

[3] Bose-Einstein Condensation, dited by A. Griffin, D. W. Snoke and S. Stringari, Cambridge University Press, 1995.

[4] K. Huang, Statistical Mechanics, $2^{\text {th }}$ Ed., John Wiley \& Sons, New York, 1987.

[5] L. Deng, E. W. Hagley, J. Wen, et al., Nature 398, 218 (1999).

S. Inouye, A. P. Chikkatur, D. M. Stamper-Kurn, et al., Science 285, 571 (1999).

M. Kozuma, Y. Suzuki, Y. Torii, et al., Science 287, 2309 (1999).

S. Inouye, T. Pfau, S. Gupta, et al., Nature 402, 641 (1999).1 
[6] M. O. Mewes, M. R. Andrews, D. M. Kurn, et al., Phys. Rev. Lett. 78, 582 (1997).

B. P. Anderson and M. A. Kasevich, Science 282, 1686 (1998).

I. Bloch, T. W. Hänsch and T. Esslinger, Phys. Rev. Lett. 82, 3008 (1999)

E. W. Hagley, L. Deng, M. Kozuma, et al., Science 283, 1706 (1999).

[7] V. S. Bagnato and D. Kleppner, Phys. Rev. A 44, 7439 (1991).

A. S. Parkins and D. F. Walls, Phys. Rep. 303, 1 (1998).

F. Dalfovo, S. Giorgini, L. P. Pitaevskii and S. Stringari, Rev. Mod. Phys. 71, 463 (1999).

Z. Yan, Phys. Rev. A 59, 4657 (1999).

[8] J. I. Kapusta, Phys. Rev. D 24, 426 (1981).

[9] H. E. Haber and H. A. Weldon, Phys. Rev. Lett. 46, 1497 (1981).

H. E. Haber and H. A. Weldon, Phys. Rev. D 25, 502 (1982)

[10] J. Bernstein and S. Dodelson, Phys. Rev. Lett. 66, 683 (1991).

K.M. Benson, J. Bernstein, and S. Dodelson, Phys. Rev. D 44, 2480 (1991).

[11] G. Petiau, University of Paris thesis (1936). Published in Acad. Roy. de Belg., Classe Sci., Mem in $8^{\circ} \mathbf{1 6}$, No. 2 (1936).

R. J. Duffin, Phys. Rev. 54, 1114 (1938).

N. Kemmer, Proc. Roy. Soc. A 173, 91 (1939).

[12] R. A. Krajcik and M. M. Nieto, Am. J. Phys. 45, 818 (1977).

[13] E. Friedman and G. Kalbermann, Phys. Rev. C 34, 2244 (1986).

[14] B. C. Clark et al, Phys. Rev. Lett. 55, 592 (1985).

[15] R. E. Kozack et al Phys. Rev. C 40, 2181 (1989).

[16] V. Gribov, Eur. Phys. J. C 10, 71 (1999).

[17] I. V. Kanatchikov, Rep. Math. Phys. 46, 107 (2000).

[18] J.T. Lunardi, L.A. Manzoni, B.M. Pimentel and J.S. Valverde, Int. J. Mod. Phys. A 17, 205 (2002).

[19] M. de Montigny, F. C. Khanna, A. E. Santana, E. S. Santos, J. D. M. Vianna, J. Phys. A 33, L273 (2000).

[20] L. K. Kerr, B. C. Clark, S. Hama, L. Ray and G. W. Hoffmann, Prog. Theor. Phys. 103, $321(2000)$.

[21] J. T. Lunardi, B. M. Pimentel and R. G. Teixeira, in Geometrical Aspects of Quantum Fields, proceedings of the 2000 Londrina Workshop, Londrina, Brazil; edited by A. A. Bytsenko, A. E. Gonçalves and B. M. Pimentel; World Scientific, Singapore (2001), p. 111. Also available as gr-qc/9909033.

[22] V. Ya. Fainberg and B. M. Pimentel, Phys. Lett. A 271, 16 (2000).

[23] J. T. Lunardi, B. M. Pimentel and R. G. Teixeira, Gen. Rel. Grav. 34, 491 (2002).

[24] R. Casana, B. M. Pimentel, J. T. Lunardi, R. G. Teixeira, Gen. Rel. Grav. 34, 1941 (2002). 
[25] B. M. Pimentel and V. Ya. Fainberg, Theor. Math. Phys. 124, 1234 (2000).

V. Ya. Fainberg and B. M. Pimentel, Braz. J. Phys. 30, 275 (2000).

[26] C. W. Bernard, Phys. Rev. D 9, 3312 (1974).

[27] J. I. Kapusta, Finite-Temperature Field Theory, Cambridge University Press, 1989.

[28] M. Le Bellac, Thermal Field Theory, Cambridge University Press, 1996.

[29] M. B. Altaie and E. Malkawi, J. Phys. A: Math. Gen. 33, 7093 (2000).

M. B. Altaie, J. Phys. A: Math. Gen. 11, 1603 (1978).

K. Kirsten and D. J. Toms, Phys. Rev. D 51, 6886 (1995).

D. J. Toms, Phys. Rev. D 47, 2483 (1993).1 